\title{
Development of seasonal flow outlook model for Ganges-Brahmaputra Basins in Bangladesh
}

\author{
Sazzad Hossain ${ }^{1}$, Raihanul Haque Khan ${ }^{2}$, Dilip Kumar Gautum ${ }^{2}$, Ripon Karmaker ${ }^{1}$, and \\ Amirul Hossain ${ }^{1}$ \\ ${ }^{1}$ Flood Forecasting and Warning Center, Dhaka, Bangladesh \\ ${ }^{2}$ Regional Integrated Multi-hazard Early Warning System, Thailand
}

Correspondence to: Sazzad Hossain (sazz176@yahoo.com)

Published: 17 October 2016

\begin{abstract}
Bangladesh is crisscrossed by the branches and tributaries of three main river systems, the Ganges, Bramaputra and Meghna (GBM). The temporal variation of water availability of those rivers has an impact on the different water usages such as irrigation, urban water supply, hydropower generation, navigation etc. Thus, seasonal flow outlook can play important role in various aspects of water management. The Flood Forecasting and Warning Center (FFWC) in Bangladesh provides short term and medium term flood forecast, and there is a wide demand from end-users about seasonal flow outlook for agricultural purposes. The objective of this study is to develop a seasonal flow outlook model in Bangladesh based on rainfall forecast. It uses European Centre for Medium-Range Weather Forecasts (ECMWF) seasonal precipitation, temperature forecast to simulate HYDROMAD hydrological model. Present study is limited for Ganges and Brahmaputra River Basins. ARIMA correction is applied to correct the model error. The performance of the model is evaluated using coefficient of determination $\left(R_{2}\right)$ and Nash-Sutcliffe Efficiency (NSE). The model result shows good performance with $R^{2}$ value of 0.78 and NSE of 0.61 for the Brahmaputra River Basin, and $R^{2}$ value of 0.72 and NSE of 0.59 for the Ganges River Basin for the period of May to July 2015. The result of the study indicates strong potential to make seasonal outlook to be operationalized.
\end{abstract}

\section{Introduction}

Bangladesh is located at the downstream end of the three major river basins, collectively known as Ganges-BrahmaputraMeghna (GBM) basin. The total catchment area is approximately 1.6 million $\mathrm{km}^{2}$ of which only about $7.5 \%$ lies within Bangladesh and the rest, $92.5 \%$ lies in India, China, Nepal and Bhutan. It is estimated that an average flow of 1009000 million $\mathrm{m}^{3}$ passes through these river systems during the monsoon season and monsoon precipitation is the major source flow (FFWC, 2014). The rivers in these three catchments mostly are originated from Himalayas mountain ranges and the pattern of flow, timing and intensity is governed by the quantity and distribution of precipitation, its form (rain or snow) and seasonality (ICIMOD, 2009). There are two distinct seasons in Bangladesh, a dry period from November to May and the wet from June to October. Over
$80 \%$ of the rainfall occurs during the monsoon or rainy season also known as flood season (WMO/GWP, 2003). The normal annual rainfall of the country varies approximately from $1200 \mathrm{~mm}$ in the west to over $5000 \mathrm{~mm}$ in the east (BWDB, 2011). Flood is a perennial problem in Bangladesh which causes huge economic losses each year. There are various factors, which contribute to the flooding in Bangladesh. One of the important factors is $80 \%$ of the country is floodplain with $50 \%$ of the country having elevation below $5 \mathrm{~m}$ of MSL. In a normal year, roughly $20 \%$ of the country is inundated, while in years of severe flooding $60-70 \%$ of the country gets affected by flood water. For instance, the duration of flood in 1998 was more than two months and $68 \%$ of the country was under water (FFWC, 2004). High population density and extreme poverty aggravates the flood situation. Moreover, the country is also affected by hydrological drought from prevailing low flow in the river systems dur- 
Table 1. Catchment Area of Brahmaputra and Ganges River.

\begin{tabular}{lrrrrrr}
\hline \multirow{2}{*}{ River Basin } & Total catchment area $\left(\mathrm{km}^{2}\right)$ & \multicolumn{5}{c}{ Catchment Area $\left(\mathrm{km}^{2}\right)$} \\
\cline { 3 - 6 } & & India & Nepal & Bhutan & China & Bangladesh \\
\hline Brahmaputra & 552000 & 195000 & - & 47000 & 270900 & 39100 \\
Ganges & 1087300 & 860000 & 147480 & - & 33520 & 46300 \\
\hline
\end{tabular}

ing the dry period. Thus, temporal variation in river flows has a significant impact on the different water usages such as irrigation, urban water supply, navigation, in stream flow requirement etc. Seasonal flow outlooks can therefore play big role in agricultural planning, selection of cropping pattern, irrigation scheduling and other areas like disaster management and longer-term water management planning.

There are some initiatives to predict medium to long range flow using climate data. Flood Forecasting and Warning Centre (FFWC) in Bangladesh issues 10 days probabilistic forecast at 38 locations along the major river systems using ECMWF medium range weather forecast (FFWC, 2013) with support from RIMES. Webster and Carlos (2004) predicted river discharge on 15-30-day time scale in the Brahmaputra and Ganges Basin in Bangladesh using ECMWF data. Seasonal outlooks for rainfall are available over the South Asian region under the South Asian Climate Outlook Forum (SASCOF) and the Bangladesh Meteorological Department also issues monthly outlook for the rainfall. Performance of longer range hydrological forecast (e.g. seasonal scale) largely depends on the accuracy of seasonal rainfall forecast. Hydrological modelling is used to generate flow using various meteorological inputs including rainfall, temperatures, soil moisture and evapotranspiration. ECMWF seasonal scale meteorological forecasts are an excellent source of forecast dataset to develop hydrological models. This study focuses on application of HYDROMAD model using ECMWF seasonal rainfall, temperature forecast data to generate flow for two large river basins- Brahmaputra and Ganges. The present study uses the mean of 41 set of ensembles available in the ECMWF seasonal forecast data set. This study also highlights potential dissemination aspects of seasonal flow outlook among the water users in Bangladesh.

\section{Study area}

The Ganges and Brahmaputra Basins are located approximately between latitude $21-31^{\circ} \mathrm{N}$ and longitude $73-96^{\circ} \mathrm{E}$. The study area consists of two big river basins which are known as Brahmaputra and Ganges. Table 1 shows individual area in square $\mathrm{km}$ of these two river basins and corresponding area in respective co-riparian countries. The majority of the Ganges Basin is located inside India and major part of the Brahmaputra Basin is situated within China. The Ganges originates from the western side of the Himalayas,

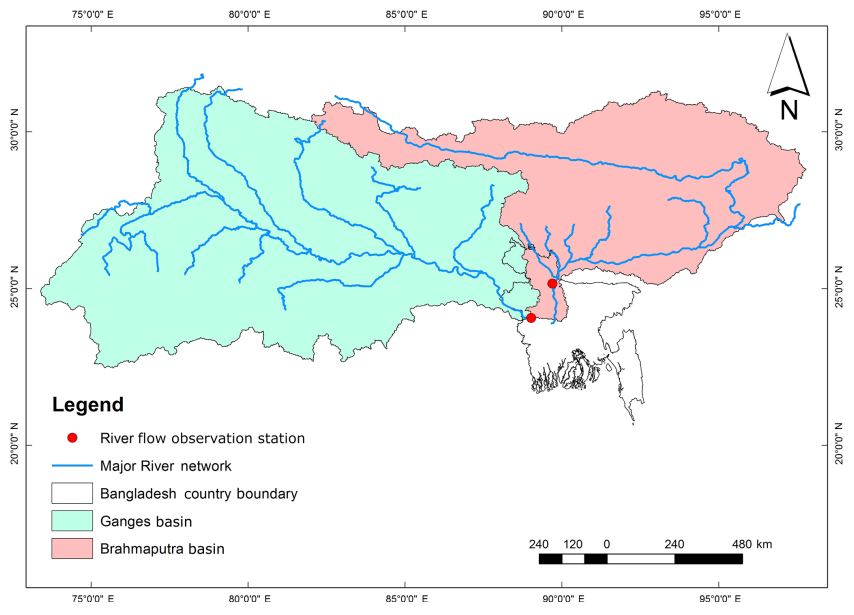

Figure 1. Ganges-Brahmaputra Basin map with respect to Bangladesh.

and it flows approximately $2000 \mathrm{~km}$ through India. It enters in Bangladesh from the western side of the country and flows towards southeast for about $250 \mathrm{~km}$ to meet confluence with the Brahmaputra. The Brahmaputra originates on the Chinese side of the Himalayas and flows about $1800 \mathrm{~km}$ through Tibet and India to enter Bangladesh from the northern part of the country. It flows for $275 \mathrm{~km}$ inside Bangladesh to join with the Ganges (Islam, 2002). Figure 1 shows the position of the Basins with respect to Bangladesh. The two river basins have distinct hydrological characteristics. Flood hydrograph of the Ganges shows that the flow starts to recede from at the end of September every year and minimum flow prevails in the river between March and April. Flow of Brahmaputra river starts rising in March due to melting of snow in the Himalayas while discharge of Ganges rises in July with the onset of monsoon. Usually, the flood flow of the Brahmaputra reaches peak in July and August, while the peaks of the Ganges occur in August and September. Though the two basins usually receive flood in two different time, synchronization of flow peaks are not uncommon and such cases cause major floorings in the country.

\section{Data and methodology}

The HYDROMAD model needs rainfall, temperature and potential evaporation to simulate model and observed flow 


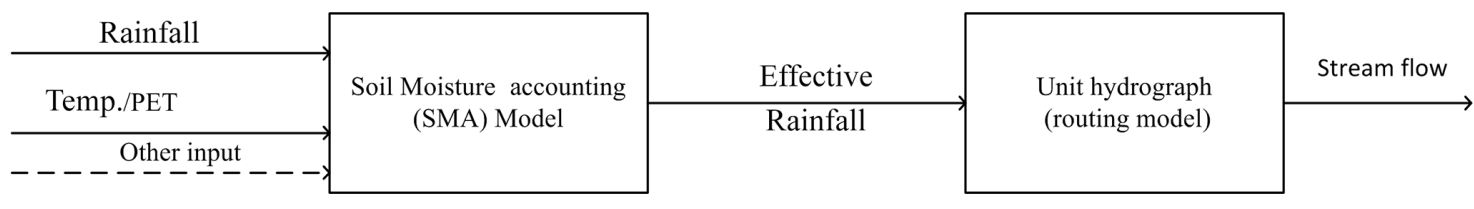

Figure 2. The modelling framework in the HYDROMAD package.
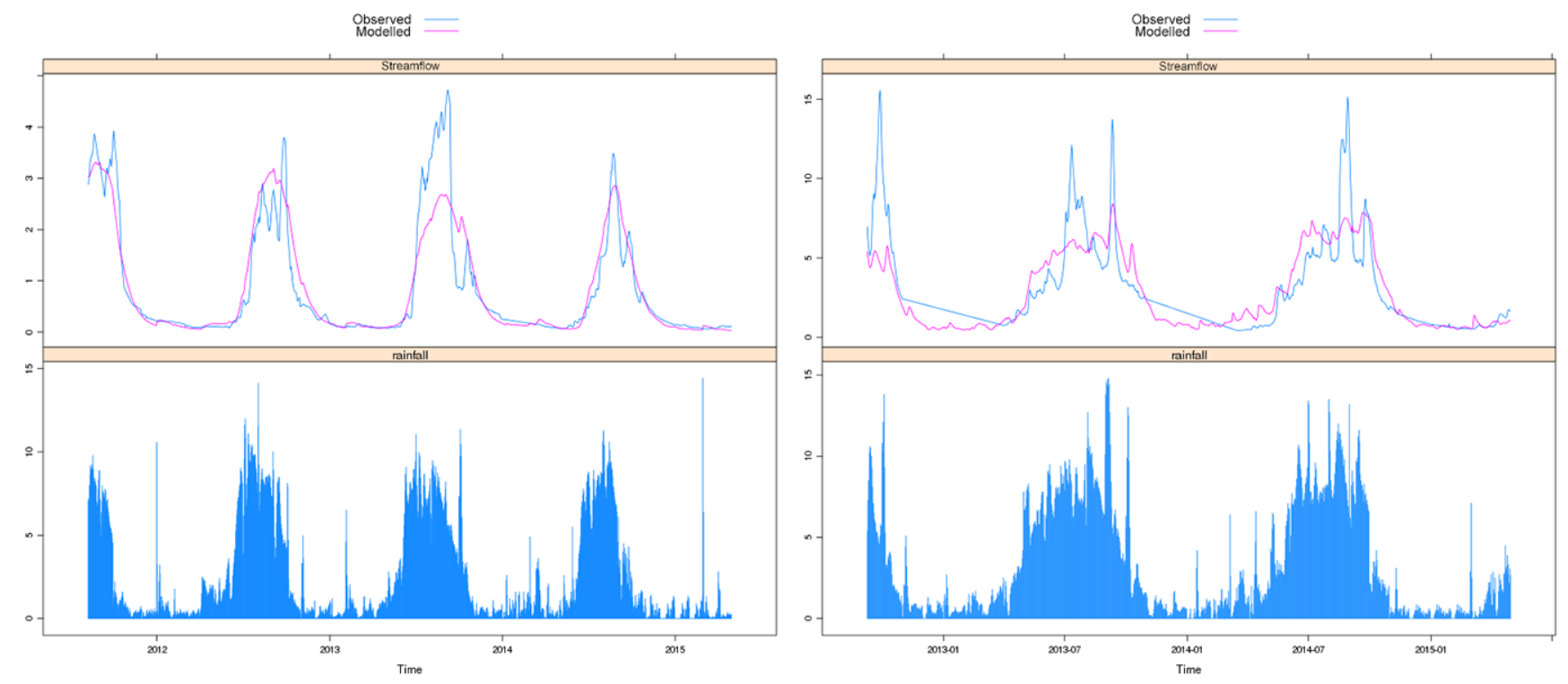

Figure 3. Observed and simulated discharge of without correction (Ganges-left and Brahmaputra-right).

data for calibration. The present study utilizes ECMWF seasonal ( 3 months and 6 months) rainfall and temperature forecast data for Ganges and Brahmaputra Basins. ECMWF seasonal forecast is ensemble forecast with 41 members, and it is the output of Atmospheric, Wave and Ocean models (Persson and Grazzini, 2011). The observed river discharge of the two basins has been taken from Bangladesh Water Development Board (BWBD) for calibration and validation. The HYDROMAD is a " $R$ " language package for hydrological modelling and related data analysis. HYDROMAD is a spatially lumped model and adopts an empirical approach. It does not explicitly represent spatial variation over the catchment area (Andrews et al., 2011). The model can predict stream flow from time series of areal rainfall and temperature or potential evapotranspiration. The modelling framework in the HYDROMAD package is based on a two-component structure: (a) a soil moisture accounting (SMA) module; and (b) a routing or unit hydrograph module. Figure 2 explains the modelling frame framework of HYDRMAD Model. SMA model converts rainfall and temperature into effective rainfall - the amount of rainfall, which eventually reaches the catchment outlet as stream flow. The routing module then converts the effective rainfall into stream flow, which amounts to defining the peak response and shape of the recession curve. The major steps in developing hydrological model are to extract seasonal ensemble forecast of rainfall and temperature for the Ganges and Brahmaputra basins and compute ensemble mean for each grid, compute Mean Areal Precipitation (MAP) and Mean Areal Temperature (MAT) over the catchment. HYDROMAD model is simulated for calibration and validation period to generate seasonal flow outlook. Statistical criteria $R^{2}$, NSE have been calculated to test model performance. ARMA correction has been applied for the best fitting of the simulated data with the observed data set. Finally, a Web Based Decision Support System has been developed to disseminate the outputs to the water managers.

\section{Result and discussion}

The model was run from 2012 to 2014 as a calibration period, and the results are presented in Fig. 3. The results shows observed peaks both of the river basins are higher than the simulated value, which indicates that the model underestimated the peak flow. However, low flows simulated by the model have a better agreement with the observed data. The reasons for the difference in observed and simulated discharge could be due to uncertainly in rainfall forecast, the inconsistency in the observed flow data used for calibration etc. ARMA correction is applied for the purpose of improving the model performance. After applying correction, peak discharge as well 

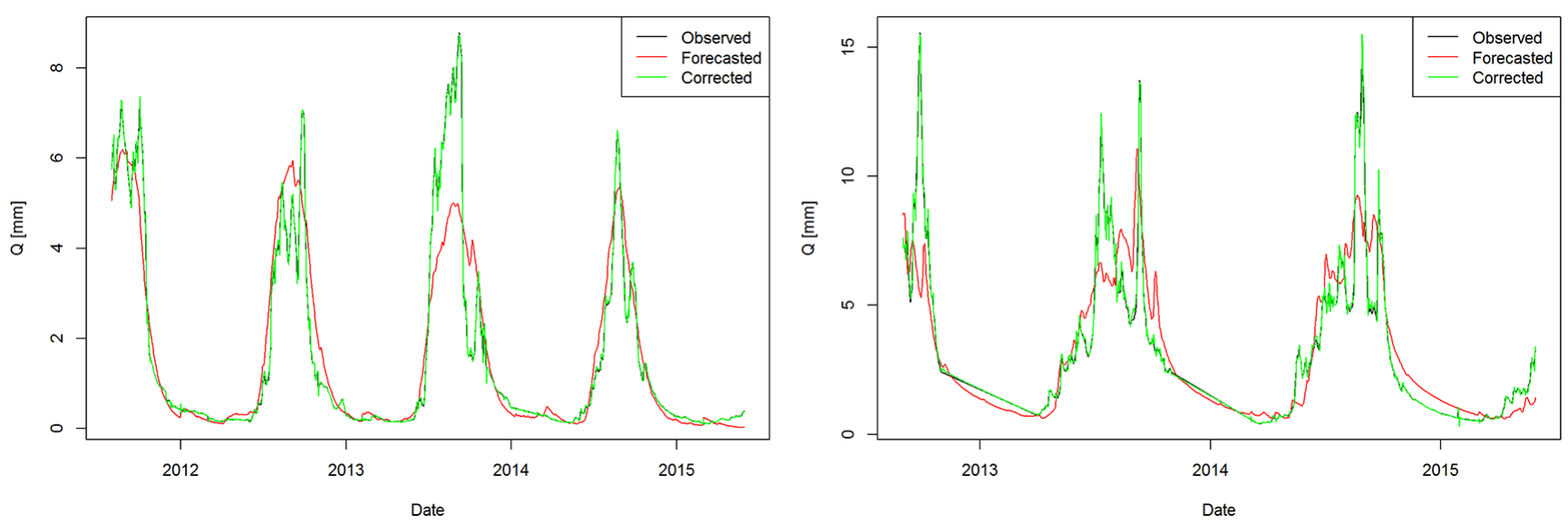

Figure 4. Observed and simulated discharge of with ARMA correction (Ganges-left and Brahmaputra-right).

as low flow, both of river basins show well matched with the observed data. Figure 4 shows hydrograph of observed and simulated discharge of Brahmaputra and Ganges after applying correction. The model performance is determined using coefficient of determination $\left(R^{2}\right)$ and Nash-Sutcliffe efficiency (NSE) for both the basins. The model has been run since January 2015 for trail operation and $R^{2}$ and NSE has been calculated to check the model performance. The result shows good agreement between observed and simulated data, with $R^{2}$ value of 0.78 and NSE of 0.61 for the Brahmaputra River Basin, and $R^{2}$ value of 0.72 and NSE of 0.59 for the Ganges River Basin for the period of May to July 2015.

An initiative has been taken to disseminate seasonal flow outlook through a web based (http://sffs.rimes.int) system. Dissemination includes average seasonal flow, normal flow and forecasted flow with three distinct colours. The dissemination system automatically generates an outlook like "The flow of Ganges River is going to be below normal for the months of September, October and November". An emailing system has been embedded into the portal to disseminate the outlook to desired stakeholders.

\section{Conclusions}

The model has been simulated for a period of 2012 to 2014 for calibration and validation purposes and based on the results, is being simulated regularly with the outputs published to a website in every month. The first phase of the study was concluded in 2015, and the overall result is very promising in terms of the application of ECMWF precipitation forecast in developing seasonal river flow outlook across the GBM basin in Bangladesh. As rainfall forecast is affected by various climate drivers so, it is important to consider those factors for forecasting with hydrological models. At present, the model forecast output is experimentally disseminated through a web-based system to get feedback from different users specially water resources planners and disas- ter managers. The Ganges-Brahmaputra is a transboundary river basin and details upstream information is not available. HYDROMAD is a lump model and takes only few data to simulate the model. So, it might be a better approach to operate for seasonal flow model to incorporate more information of upper basin as well. Assessment and research on the application of seasonal flow outlook in different field of water resources management like irrigation, agriculture, disaster management etc. should be carried out to make this forecast meaningful.

\section{Data availability}

Hydrological data of the Ganges and Brahmaputra basin inside Bangladesh is accessible from http://www.hydrology. bwdb.gov.bd/. The observed rainfall data also available from Bangladesh Metrological Department (BMD) with special request, and ECMWF seasonal rainfall forecast available from Regional Integrated Multi-Hazard Early Warning System (RIMES) taken Result of the present study also available from http://sffs.rimes.int.

Acknowledgements. The present research work has been conducted for the increase of flood forecast lead-time of Bangladesh by the technical support of Regional Integrated Multi-hazard Early Warning System.

\section{References}

Andrews, F. T., Croke, B. F. W., and Jakeman, A. J.: An open software environment for hydrological model assessment and development, Environ. Modell. Softw., 26, 1171-1185, doi:10.1016/j.envsoft.2011.04.006, 2011.

BWDB: Inception Report on Research and Prediction Modelling Through Upgrading of Flood Forecasting System by Increasing Lead Time, BWBD, Dhaka, 30, 2011. 
FFWC: Annual Flood Report 2004, Flood Forecasting and Warning Center, BWBD, Dhaka, 6, 2004.

FFWC: Annual Flood Report 2013, Flood Forecasting and Warning Center, BWBD, Dhaka, 70, 2013.

FFWC: Annual Flood Report 2014, Flood Forecasting and Warning Center, BWBD, Dhaka, 1, 2014.

ICIMOD: Project Proposal on The Hindu Kush-Himalayan Hydrological Cycle Observing System, ICIMOD, Kathamundu, Nepal, $1,2009$.

Islam, S.: Hydrology of Floods in South Asia, SaciWATERs Workshop on Floods in South Asia, Dhaka, Bangladesh, 28-30 November, 2002.
Persson, A. and Grazzini, F.: User guide to ECMWF forecast products, Version 4.0, available at: http://www.ecmwf.int (last access: 15 April 2015), 2011.

Regional Integrated Multi-Hazard Early Warning System (RIMES): Bangladesh Seasonal Flow Outlook, available at: http://sffs. rimes.int, 2016.

Webster, P. J. and Carlos, H.: Prediction of monsoon rainfall and river discharge on 15-30-day Time scales, American meteorological society, 85, 1745-1765, 2004.

WMO/GWP: Integrated Flood Management Case Study Bangladesh: Flood Management, The Associated Programme on Flood Management, 1, 2003. 